\title{
Nonadiabatic Approach to Spin-Peierls Transitions via Flow Equations
}

\author{
Götz S. Uhrig \\ Institut für Theoretische Physik, Universität zu Köln, Zülpicherstraße 77, 50937 Köln, Germany.
}

\begin{abstract}
The validity of the adiabatic approach to spin-Peierls transitions is assessed. An alternative approach is developed which maps the initial magneto-elastic problem to an effective magnetic problem only. Thus the equivalence of magneto-elastic solitons and magnetic spinons is shown. No soft phonon is required for the transition. Temperature dependent couplings are predicted in accordance with the analysis of experimental data.

75.10.Jm, 63.20.Kr, 63.20.Ls
\end{abstract}

Around 1980 there was a great interest in the phenomenon of spin-Peierls (SP) transitions [1] where the coupling of lattice degrees of freedom to quasi onedimensional $(d=1)$ magnetic degrees of freedom leads to a phase transition into a dimerized phase. This interest has been vividly renewed recently due to the discovery of the first inorganic SP substance $\mathrm{CuGeO}_{3}$ [2, 3].

The instability of the coupled spin-phonon system towards dimerization results from the susceptibility of the magnetic $d=1$ subsystem towards $2 k_{\mathrm{F}}$ perturbations, i.e. dimerization. The gain in magnetic energy due to dimerization overcompensates the loss in elastic energy of the lattice distortion. The present work is motivated by the wealth of information available for the SP transition in $\mathrm{CuGeO}_{3}$.

The theoretical picture of SP transitions has been developped in a number of articles (e.g. [4 10]). It relies so far mostly on an adiabatic treatment of the phonons. Cross and Fisher discussed this point most comprehensively [6]. By random phase approximation (RPA) they investigate the stability of the uniform phase. The RPA is the consistent extension of the mean-field treatment on the one-particle level to the two-particle level of susceptibilities. Cross and Fisher's point is the importance of a "pre-existing soft phonon". This means that the phonons responsible for the distortion have a low energy already before the interaction with the correlated system is taken into account. Moreover, they require that the phonon dispersion perpendicular to the chain direction is very large. Thus the lattice prefers that whole planes perpendicular to the correlated chains move coherently so that the moving objects are heavy.

The reason for the above requirements is that the mean-field approach is appropriate if the fluctuations are small compared to the expectation value. This is the case if the distortions are made up by a large number of phonons which in turn means that the phonon energies must be small. The phonons must be slow and heavy. Then it is plausible to consider the phonon subsystem as the slow subsystem which is renormalized by the fast magnetic degrees of freedom. In this picture, the SP transition is signalled by the vanishing of a renormalized phonon frequency [6].

Whereas the experimental data for organic SP substances such as (TTF) $\mathrm{CuS}_{4} \mathrm{C}_{4}\left(\mathrm{CF}_{3}\right)_{4}$ supports the RPA approach $\|6\|$, the experimental evidence for the inorganic $\mathrm{CuGeO}_{3}$ points into the opposite direction [11]. Braden et al. found that two out of four optical phonons allowed by symmetry contribute appreciably to the distortion in a ratio of $3: 2$ [11]. The more important phonon is at $6.8 \mathrm{Thz}(330 \mathrm{~K})$; its dispersion at the zone boundary is essentially flat decreasing towards the zone centre to $\approx 3 \mathrm{THz}$. The other phonon is at $3.2 \mathrm{THz}(150 \mathrm{~K})$ and practically dispersionsless. The magnetic exchange coupling $J$ lies in the range $115 \mathrm{~K}$ to $160 \mathrm{~K}$ [12 16]. It is evident that the assumption of a pre-existing soft phonon is inadequate. Things get even worse if one takes into consideration the results for a XY chain with $d=1$, Einstein phonons [17] which show that the phonon energy $\omega$ must be small compared to the resulting gap $\Delta$ for the adiabatic approach to be reasonable. The condition $\omega<\Delta$, however, is definitely not fulfilled in $\mathrm{CuGeO}_{3}$ with $\Delta=23 \mathrm{~K}$ [18]. In view of these facts it is not astounding that so far no phonon softening at the SP transition was found experimentally. For comparison, we recall the numbers for the best-known substance (TTF) $\mathrm{CuS}_{4} \mathrm{C}_{4}\left(\mathrm{CF}_{3}\right)_{4}$ with soft phonon: $J=68 \mathrm{~K}$, $T_{\mathrm{SP}}=12 \mathrm{~K}\left(\Rightarrow \Delta \approx 1.77 T_{\mathrm{SP}} \approx 20 \mathrm{~K}\right), \omega \approx 10 \mathrm{~K}$ where $\omega<\Delta$ is fulfilled [1].

In the present work we develop a route to Peierls transitions not based on the assumption of phononic adiabaticity. Results of previous works [19 21] on nonadiabaticity will be extended. We will view the phonon subsystem as fast and the spin subsystem as slow. The unperturbed spin system is always gapless, i.e. the magnetic subsystem always has low-lying excitations well below any (optical) phonon energy. These low-lying excitations are influenced most by the interaction of phonons and spins. To them the phonons are fast. So we treat the phonons as quickly adapting and derive an effective dressed spin model. Pytte did the same for an Ising model which allowed the rigorous elimination of phonons [19] stressing already the importance of avoiding a meanfield approximation for the displacements.

Technically we use the recently developed flow equation approach to treat the spin-phonon system 22]. The idea is to rotate away the direct interaction with phonons similar to what is done in Fröhlich's approach 23. This approach has been improved considerably by Lenz and 
Wegner for electron-phonon interactions 24]. In the improved version the generated effective couplings are by far less singular than in Fröhlich's approach.

The flow equation approach "diagonalizes" a Hamiltonian in a continuous unitary transformation parametrized by $l \in[0, \infty]$. This means $H(0)$ is the bare Hamiltonian as given and $H(\infty)$ is the resulting (more) diagonal Hamiltonian. The unitary transformation is defined by its antihermitean infinitesimal generator $\eta(l)$ via

$$
\frac{d H}{d l}=[\eta(l), H(l)] .
$$

A good choice for $\eta$ is

$$
\eta=\left[H_{\mathrm{D}}(l), H(l)\right]
$$

where $H_{\mathrm{D}}$ is the suitably chosen diagonal Hamiltonian [22]. The main feature of $\eta$ as defined in (2) is that it respects the idea of renormalization in that it eliminates first matrix elements connecting very different energies [25].

The SP system we consider is given by the Hamiltonian

$$
\begin{aligned}
H & =H_{\mathrm{S}}+H_{\mathrm{B}}+H_{\mathrm{SB}} \quad H_{\mathrm{S}}=\sum_{\vec{q}} d(\vec{q}) \vec{S}_{\vec{q}} \vec{S}_{-\vec{q}} \\
H_{\mathrm{B}} & =\sum_{\vec{q}} \omega(\vec{q}) b_{\vec{q}}^{+} b_{\vec{q}} \quad H_{\mathrm{SB}}=\sum_{\vec{q}} A_{\vec{q}}\left(b_{\vec{q}}^{+}+b_{-\vec{q}}\right) \\
A_{\vec{q}} & =\sum_{\vec{k}} g(\vec{q}, \vec{k}) \vec{S}_{\vec{k}} \vec{S}_{-\vec{k}-\vec{q}} .
\end{aligned}
$$

in obvious notation in momentum space. Note that according to [22] $A_{\vec{q}}$ should be normal-ordered $A_{\vec{q}} \rightarrow$ $A_{\vec{q}}-\left\langle A_{\vec{q}}\right\rangle$. The particular choice (before eq. 12 and eq. 14) for $A_{\vec{q}}$ will circumvent this problem. We assume inversion symmetry so that $\omega(\vec{q})=\omega(-\vec{q})$ and $d(\vec{q})=d(-\vec{q})$. Hermiticity requires $A_{-\vec{q}}^{+}=A_{\vec{q}}$ or equivalently $g^{*}(-\vec{q},-\vec{k})=g(\vec{q}, \vec{k})$. The linear boson terms become $l$-dependent for the unitary transformation

$$
H_{\mathrm{SB}}(l)=\sum_{\vec{q}}\left(T_{\vec{q}}(l) b_{\vec{q}}^{+}+T_{\vec{q}}^{+}(l) b_{\vec{q}}\right)
$$

with the starting condition $T_{\vec{q}}(0)=A_{\vec{q}}$. The objective of the unitary transformation is to disentangle phonons and spins. Thus we choose $H_{\mathrm{D}}=H_{\mathrm{S}}+H_{\mathrm{B}}$. To leading order in $g$ we do not need to consider a possible $l$-dependence of $H_{\mathrm{D}}$ since the $l$-dependent terms enter only in order $g^{2}$ as we will see. These induced terms of order $g^{2}$ and higher lead to a new contribution $\Delta H$ to the Hamiltonian. Introducing the Liouville operator $\mathcal{L}$ for the commutation with $H_{\mathrm{S}}: \mathcal{L} A:=\left[H_{\mathrm{S}}, A\right]$ we choose for the generator $\eta$

$$
\begin{aligned}
& \eta(l)=\left[H_{\mathrm{D}}, H_{\mathrm{SB}}\right] \\
& =\sum_{\vec{q}}\left((\mathcal{L}+\omega(\vec{q})) T_{\vec{q}}(l) b_{\vec{q}}^{+}+(\mathcal{L}-\omega(\vec{q})) T_{\vec{q}}^{+}(l) b_{\vec{q}}\right)
\end{aligned}
$$

which is motivated by (2). The flow equation (11) leads to

$$
\begin{aligned}
& \frac{d H}{d l}=\left[\eta, H_{\mathrm{SB}}\right]+[\eta, \Delta H] \\
& -\sum_{\vec{q}}\left((\mathcal{L}+\omega(\vec{q}))^{2} T_{\vec{q}}(l) b_{\vec{q}}^{+}+(\mathcal{L}-\omega(\vec{q}))^{2} T_{\vec{q}}^{+}(l) b_{\vec{q}}\right)
\end{aligned}
$$

In linear order in $g$ we have to fulfil the flow equation

$$
\frac{d T_{\vec{q}}}{d l}=-(\mathcal{L}+\omega(\vec{q}))^{2} T_{\vec{q}}
$$

which is formally solved by

$$
T_{\vec{q}}(l)=\exp \left(-(\mathcal{L}+\omega(\vec{q}))^{2} l\right) A_{\vec{q}} .
$$

Based on (8) the additional Hamilton part $\Delta H$ can be calculated

$$
\begin{aligned}
& \frac{d \Delta H}{d l}=\left[\eta, H_{\mathrm{SB}}\right]+\mathcal{O}\left(g^{3}\right) \\
& =-\sum_{\vec{q}, \vec{k}}\left[T_{\vec{q}} b_{\vec{q}}^{+}+T_{\vec{q}} b_{\vec{q}}, D_{\vec{q}} b_{\vec{q}}^{+}-D_{\vec{q}}^{+} b_{\vec{q}}\right]+\mathcal{O}\left(g^{3}\right) \\
& =-\sum_{\vec{q}}\left(D_{\vec{q}}^{+} T_{\vec{q}}+T_{\vec{q}}^{+} D_{\vec{q}}\right) \\
& \quad+\sum_{\vec{q}, \vec{k}}\left(b_{\vec{q}}^{+} b_{\vec{k}}^{+}\left[D_{\vec{q}}, T_{\vec{k}}\right]+\text { h.c. }\right) \\
& \quad+\sum_{\vec{q}, \vec{k}}\left(b_{\vec{q}}^{+} b_{\vec{k}}\left(\left[T_{\vec{q}}, D_{\vec{k}}^{+}\right]+\left[D_{\vec{q}}, T_{\vec{k}}^{+}\right]\right)\right)+\mathcal{O}\left(g^{3}\right)
\end{aligned}
$$

where we used the shorthand $D_{\vec{q}}=(\mathcal{L}+\omega(\vec{q})) T_{\vec{q}}$.

To obtain from (9) an effective spin Hamiltonian we use a mean-field approach and replace the quadratic boson terms by its expectation values. This is absolutely systematic in the sense of an expansion in $g$. Taking the expectation values neglects fluctuation effects of the order $g^{2}$ due to the interaction. But since the two-boson terms appear only as $g^{2}$-terms the total error due to the mean-field treatment is of the order $g^{4}$. Applying the same mean-field approach to the unspecified $g^{3}$ terms annihilates them because they contain necessarily an odd number of boson operators. Thus the effective spin model is exact up to $\mathcal{O}\left(g^{4}\right)$.

Replacing $b_{\vec{q}}^{+} b_{\vec{k}}$ by $\delta_{\vec{q}, \vec{k}}(\exp (\omega(\vec{q}) / T)-1)^{-1}$ and omitting the terms in (9d) we obtain finally

$$
\begin{aligned}
& \frac{d \Delta H}{d l}=\sum_{\vec{q}}\left(X_{\vec{q}}+\operatorname{coth}\left(\frac{\omega(\vec{q})}{2 T}\right) Y_{\vec{q}}\right) \quad \text { with } \\
& X_{\vec{q}}=-\frac{1}{2}\left(D_{\vec{q}} T_{\vec{q}}^{+}+D_{\vec{q}}^{+} T_{\vec{q}}+T_{\vec{q}} D_{\vec{q}}^{+}+T_{\vec{q}}^{+} D_{\vec{q}}\right) \\
& Y_{\vec{q}}=\frac{1}{2}\left(\left[T_{\vec{q}}, D_{\vec{q}}^{+}\right]+\left[D_{\vec{q}}, T_{\vec{q}}^{+}\right]\right) .
\end{aligned}
$$

From (10) the Hamiltonian corrections $\Delta H_{X / Y}$ are found by integration over $l$ and summation over $\vec{q}: \Delta H_{X}=$ $\int_{0}^{\infty} \sum_{\vec{q}} X_{\vec{q}} d l$ and $\Delta H_{Y}=\int_{0}^{\infty} \sum_{\vec{q}} \operatorname{coth}(\omega(\vec{q}) /(2 T)) Y_{\vec{q}} d l$. 
In order to get an impression of what (10) means we assume $J \ll \omega(\vec{q})$ and calculate the leading contributions in $\mathcal{L}$ to $X_{\vec{q}}$ (even in $\mathcal{L}$ ) and $Y_{\vec{q}}$ (odd in $\mathcal{L}$ ). After some algebra and integration we find

$$
\begin{aligned}
\Delta H_{X} & =-\sum_{\vec{q}} \frac{1}{\omega(\vec{q})} A_{\vec{q}}^{+} A_{\vec{q}}=\frac{-1}{\omega} \sum_{i} A_{i}^{+} A_{i} \\
\Delta H_{Y} & =\frac{1}{2} \sum_{\vec{q}} \frac{1}{\omega^{2}(\vec{q})} \operatorname{coth}\left(\frac{\omega(\vec{q})}{2 T}\right)\left[A_{\vec{q}}^{+}, \mathcal{L} A_{\vec{q}}\right] \\
& =\frac{1}{2 \omega^{2}} \operatorname{coth}\left(\frac{\omega}{2 T}\right) \sum_{i}\left[A_{i}^{+}, \mathcal{L} A_{i}\right]
\end{aligned}
$$

where we simplified the formulae $(11 \mathrm{a})$ and $(11 \mathrm{~d})$ in real space one step further approximating the phonons by Einstein phonons. The term $\Delta H_{X}$ corresponds to the results obtained previously by other methods 19 21. To the author's knowledge, the $T$-dependent term $\Delta H_{Y}$ has not yet been described. The result of Pytte [19] is found back by observing that in the Ising model $\mathcal{L} A_{i}$ vanishes since all terms involving only $S^{z}$ commute, i.e. $\Delta H_{Y}$ becomes zero. The result in 21] is retrieved on observing that $g^{2} / \omega$ is proportional to $J^{2} /\left(m \omega^{2}\right)$ in 21] since the displacments equal $u_{i}=\left(b_{i}+b_{i}^{\dagger}\right) / \sqrt{2 m \omega}$. Neglecting the phononic kinetic energy while keeping their potential one constant corresponds to the limit $m \rightarrow 0$ with $m \omega^{2}$ constant, i.e. $\omega \rightarrow \infty$. So the $g^{2} / \omega$ term is constant and kept while terms $g^{2} \mathcal{O}\left(\omega^{-2}\right)$ like $\Delta H_{Y}$ are neglected in 21]. To further enhance the plausibility of the result (11) we note that it equals the result one gets by Fröhlich's method 233 in the two leading orders $g^{2} / \omega$ and $g^{2} J / \omega^{2}$. The difference of the flow equation approach and Fröhlich's approach appears only in the $1 / \omega^{3}$ terms coming from (10). Insofar Fröhlich's approach can also be used to derive (11). The flow equation approach, however, is a better starting point for future higher-order calculations in $g / \omega$ and $J / \omega$ which take $\ell$-dependent couplings into account. This is the reason why this method is chosen here.

Specifically, we consider first strictly one dimensional phonons $A_{i}=g\left(\vec{S}_{i} \vec{S}_{i+1}-\vec{S}_{i} \vec{S}_{i-1}\right)$. This choice guarantees $\left\langle A_{i}\right\rangle=0$ in the symmetry unbroken phase so that $A_{i}$ is normal-ordered. With this $A_{i}$ we have

$$
\Delta H_{X}=\frac{g^{2}}{\omega} \sum_{i}\left(\vec{S}_{i} \vec{S}_{i+1}+\frac{1}{2} \vec{S}_{i} \vec{S}_{i+2}-\frac{3}{8}\right) .
$$

For $\Delta H_{Y}$ we have to know $d(\vec{q})$ in (3a). We assume nearest and next-nearest neighbour interaction $J$ and $\alpha J$, respectively: $d(\vec{q})=J\left(\cos \left(q_{1}\right)+\alpha \cos \left(2 q_{1}\right)\right)$. One obtains

$$
\begin{aligned}
& \Delta H_{Y}=\frac{J}{4} \frac{g^{2}}{\omega^{2}} \operatorname{coth}\left(\frac{\omega}{2 T}\right) \times \\
& \sum_{i}\left(-(3-3 \alpha) \vec{S}_{i} \vec{S}_{i+1}+(3-5 \alpha) \vec{S}_{i} \vec{S}_{i+2}+2 \alpha \vec{S}_{i} \vec{S}_{i+3}\right)
\end{aligned}
$$

where products with four different spins are omitted.
Even if no frustration is present in the original model $(\alpha=0)$ the dressing of the spins with phonons induces frustration $\alpha_{\text {eff }}>0$. The couplings are temperature dependent since they are mediated by the phonons. Using the term "spinon" for a purely magnetic elementary $S=$ $1 / 2$ excitation and the term "soliton" for the joint magnetic and elastic $S=1 / 2$ excitation we state that the solitons of the Hamiltonian (3) are unitarily equivalent to the spinons of the Hamiltonian $H=H_{\mathrm{S}}+H_{\mathrm{B}}+\Delta H_{X}+\Delta H_{Y}$. This shows that solitons and spinons are in essence the same entity and puts Affleck's supposition in this respect [26] on a quantitative basis.

The low-lying excitations of the frustrated Heisenberg chain are spinons which are gapless for $\alpha_{\text {eff }}<\alpha_{\mathrm{c}}=0.241$ 27 and gapful above $\alpha_{\mathrm{c}}$ 28 where the system undergoes also a spontaneous symmetry breaking of the translational symmetry towards a dimerized phase. The continuum starts right at the gap energy [29]. These facts imply that a single chain shows a SP transition only above a certain value of the interaction in contrast to the results of the adiabatic treatment (see also [17]). Furthermore, no "double gap" feature 30] occurs in a single chain.

An elastic interchain coupling in a chain ensemble is included if the local phonons influence also neighbouring chains

$$
\begin{aligned}
A_{i, j}= & g\left(\vec{S}_{i, j} \vec{S}_{i+1, j}-\vec{S}_{i, j} \vec{S}_{i-1, j}+\right. \\
& f \sum_{<j, j^{\prime}>}\left(\vec{S}_{i, j^{\prime}} \vec{S}_{i+1, j^{\prime}}-\vec{S}_{i, j^{\prime}} \vec{S}_{i-1, j^{\prime}}\right)
\end{aligned}
$$

where $j$ is the chain index and $j$ and $j^{\prime}$ are adjacent chains. The factor $|f|<1$ indicates the influence of a certain distortion on one chain onto adjacent chains. Due to the commutators in (11d) a finite $f$ changes $\Delta H_{Y}$ in (13) only by renormalizing $g^{2} \rightarrow \tilde{g}^{2}=g^{2}\left(1+z f^{2}\right)$ where $z$ is the number of neighbouring chains each chain has. The same renormalization takes place in $\Delta H_{X}$. Additionally, terms linking different chains occur like $-\left(g^{2} f / \omega\right)\left(\vec{S}_{i, j} \vec{S}_{i+1, j}-\vec{S}_{i} \vec{S}_{i-1}\right)\left(\vec{S}_{i, j^{\prime}} \vec{S}_{i-1, j^{\prime}}-\vec{S}_{i, j^{\prime}} \vec{S}_{i-1, j^{\prime}}\right)$. These terms drive really the finite temperature SP transition since they enable at low enough temperature a coherent dimerization throughout the whole lattice. We will call these terms coherence terms. Their influence on the low-lying excitations is to confine pairs of spinons (solitons) to triplets or to singlets. For the realistic case of weak coupling $g^{2} f / \omega \ll J$ a mean-field treatment is justified. This amounts up to the treatment of dimerized, frustrated chains with self-consistently determined dimerization. Hence the confinement is the same as in dimerized chains (see e.g. 26, 31]). This explains why the adiabatic approaches based on dimerized, frustrated chains capture correctly the physics of the dimerized SP phase at low $T$.

The main difference of the fast-phonon scenario to the adiabatic one is the absence of a soft phonon at the transition. The transition is characterized by growing domains of coherent dimerization the size of which diverges 
at $T_{\mathrm{SP}}$. No renormalized phonon frequency needs to vanish. Interestingly, the RPA shows similar results in the nonadiabatic parameter regime [32].

How does this fit to the approach used so far? In the usual RPA treatment [6] the phononic self-energy contributes not only a real part but also an equally strong imaginary part which stands for strong damping [33]. Thus the real, untransformed phonons are not appropriate quasi-particles. If we transform the propagator of the real phonons in the same unitary way as the Hamiltonian we see that via $b_{i}^{\dagger} \rightarrow b_{i}^{\dagger}-\frac{g}{\omega} A_{i}+\ldots$ not only the transformed phonons matter but also $S=0$ excitations of the effective spin model. This means that the observation of the real phonons reveals not only a sharp peak at the high frequency $\omega$ but also a continuum at low frequencies of the order of $J$. The low energy continuum changes on passing through the spin-Peierls transition. In the vicinity of $T_{\mathrm{SP}}$ one expects some critical fluctuations close to zero energy. Below $T_{\mathrm{SP}}$ a gap should appear which equals twice the triplet gap or less if bound states are present [31,34. But the phonon peak is not lowered towards zero energy at the transition. This is in accordance with the results known so far for $\mathrm{CuGeO}_{3}$.

We attempt to estimate orders of magnitude for the couplings in $\mathrm{CuGeO}_{3}$. Let us assume that $T_{\mathrm{SP}}$ is of the order of $g^{2} / \omega$ then $g^{2} / \omega^{2} \approx T_{\mathrm{SP}} / \omega \approx 15 K / 150 K=0.1$ is roughly one tenth which justifies the expansion in $g^{2} / \omega^{2}$.

The extension of (11) to several phonons is straightforward. Using the phonon energies and their relative distortion as experimental input and the coupling $g$ as fit parameter it is possible to reproduce the experimental $\chi(T)$ data nearly as well as in [16] from where also the $\chi(T)$ data was taken. This shows that the assumption of $T$-dependent couplings is not ruled out by the $\chi(T)$ data. With the same parameter as for the $\chi(T)$ fit we find $J(50 \mathrm{~K})=162 \mathrm{~K}$ and $J(300 \mathrm{~K})=140 \mathrm{~K}$ which agrees very well to $J(50 \mathrm{~K})=158 \mathrm{~K}$ and $J(300 \mathrm{~K})=136 \mathrm{~K}$ deduced by Fabricius and Löw [35] from experimental $S(q, \omega)$ data. This excellent agreement confirms the validity of the approach used and in particular the prediction of $T$ dependent couplings.

In summary, we discussed the validity of the phonon adiabatic approach for SP transitions and in particular $\mathrm{CuGeO}_{3}$. The phonon adiabatic approach is inadequate for the latter system. We developed a promising alternative approach relying on the flow equation technique. Magneto-elastic solitons are mapped to magnetic spinons in an effective, purely magnetic Hamiltonian. The phonon dynamics induces a $T$-dependent frustration. No soft phonon signals the SP transition which is driven by the coherence terms in an effective magnetic model.

The author is indebted to M. Braden for making his results available to him prior to publication. He acknowledges helpful discussions with M. Braden, W. Brenig, B. Büchner, E. Müller-Hartmann, and H. J. Schulz. This work was supported by the DFG through SFB 341.
[1] J. W. Bray, L. V. Interrante, I. C. Jacobs, and J. C. Bonner, in Extended Linear Chain Compounds, edited by J. S. Miller (Plenum Press, New York, 1983), Vol. 3, p. 353.

[2] M. Hase, I. Terasaki, and K. Uchinokura, Phys. Rev. Lett. 70, 3651 (1993)

[3] J. P. Boucher and L. P. Regnault, J. Phys. I France 6, 1939 (1996)

[4] E. Pytte, Phys. Rev. B 10, 4637 (1974)

[5] J. W. Bray et al., Phys. Rev. Lett. 35, 744 (1975)

[6] M. C. Cross and D. S. Fisher, Phys. Rev. B 19, 402 (1979)

[7] M. C. Cross, Phys. Rev. B 20, 4606 (1979)

[8] T. Nakano and H. Fukuyama, J. Phys. Soc. Jpn. 49, 1679 (1980); ibid. 50, 2489 (1981)

[9] A. I. Buzdin, M. L. Kulić, and V. V. Tugushev, Solid State Commun. 48, 483 (1983)

[10] M. Fujita and K. Machida, J. Phys. Soc. Jpn. 53, 4395 (1984); ibid. J. Phys. C21, 5813 (1988)

[11] M. Braden et al., Phys. Rev. B 54, 1105 (1996); ibid. Phys. Rev. Lett. in press

[12] M. Nishi, O. Fujita, and J. Akimitsu, Phys. Rev. B 50, 6508 (1994)

[13] J. Riera and A. Dobry, Phys. Rev. B 51, 16098 (1995)

[14] G. Castilla, S. Chakravarty, and V. J. Emery, Phys. Rev. Lett. 75, 1823 (1995)

[15] G. S. Uhrig, Phys. Rev. Lett. 79, 163 (1997)

[16] K. Fabricius et al., Phys. Rev. B 57, 1102 (1998)

[17] L. G. Caron and S. Moukouri, Phys. Rev. Lett. 76, 4050 (1996)

[18] L. P. Regnault et al., Phys. Rev. B 53, 5579 (1996)

[19] E. Pytte, Phys. Rev. B 10, 2039 (1974)

[20] U. Brandt and H. Leschke, Z. Phys. 271, 295 (1974)

[21] F. H. L. Essler, A. M. Tsvelik, and G. Delfino, Phys. Rev. B 56, 11001 (1998)

[22] F. J. Wegner, Ann. Physik 3, 77 (1994)

[23] H. Fröhlich, Phys. Roy. Soc. Lond. A215, 291 (1952)

[24] P. Lenz and F. Wegner, Nucl. Phys. B 482693 (1996)

[25] S. K. Kehrein and A. Mielke, Ann. Phys. 252, 1 (1996)

[26] I. Affleck, in Dynamical Properties of Unconventional Magnetic Systems (NATO ASI, Geilo, Norway, 1997).

[27] R. Jullien and F. D. M. Haldane, Bull. Am. Phys. Soc. 28, 344 (1983); K. Okamoto and K. Nomura, Phys. Lett. A169, 433 (1992)

[28] R. Chitra et al., Phys. Rev. B 52, 6581 (1995); S. R. White and I. Affleck, Phys. Rev. B 54, 9862 (1996)

[29] B. S. Shastry and B. Sutherland, Phys. Rev. Lett. 47, 964 (1981)

[30] M. Aïn et al., Phys. Rev. Lett. 78, 1560 (1997)

[31] G. S. Uhrig and H. J. Schulz, Phys. Rev. B 54, R9624 (1996)

[32] H. J. Schulz, private communication 1998

[33] C. Gros, Korrelationstage, Dresden 1997

[34] G. Bouzerar, A. P. Kampf, and F. Schönfeld, cond/mat9701176

[35] K. Fabricius and U. Löw, cond-mat 9709160 